\title{
Influencia del modelo estético e insatisfacción corporal en adolescentes mujeres de una institución educativa estatal de Lima este, 2016
}

\author{
Influence of the aesthetic model and body dissatisfaction in adolescent females of a State educational \\ institution of Lima east, 2016
}

Jhonny Agesto Pinaud ${ }^{1}$, Betsaida Barja Becerra2* ${ }^{*}$, Aida Santillán Mejía ${ }^{3}$

\begin{abstract}
RESUMEN
Objetivo: Determinar la relación entre las influencias del modelo estético e insatisfacción corporal en adolescentes mujeres de $3^{\circ}$ a $5^{\circ}$ de secundaria de una institución educativa estatal de Lima este, 2016. Materiales y métodos: El estudio es de diseño no experimental, de corte transversal y de alcance correlacional. Para lograrlo, se contó con una muestra de 255 estudiantes mujeres cuyas edades oscilan entre 13 y 17 años, a quienes se les aplicó el Cuestionario de Influencias del Modelo Estético (CIMEC) y el Body Shape Questionnarie (BSQ). Resultados: Fueron obtenidos a través del coeficiente de correlación de Spearman muestran una relación altamente significativa entre la influencia del modelo estético y la insatisfacción corporal $\left(\mathrm{rho}=, 785^{* *}, \mathrm{p}<0,000\right)$. Asimismo, la relación entre las dimensiones de influencias del modelo estético y la variable de insatisfacción corporal son significativas. Conclusiones: A mayor influencia del modelo de belleza, mayor será la preocupación por la imagen corporal y el peso en las adolescentes.
\end{abstract}

Palabras claves: Modelos estéticos, insatisfacción corporal, adolescencia.

\begin{abstract}
Objective: To determine the relationship between the influences of the aesthetic model and body dissatisfaction in adolescent females from 3rd to 5th of high school of a state educational institution of east Lima, 2016. Materials and methods: The design of this study is non-experimental, cross sectional and of a correlational scope. To achieve this, a sample of 255 female students whose ages ranged from 13 to 17 years old, who were submitted to the Aesthetic Model Influences Questionnaire (CIMEC) and Body Shape Questionnaire (BSQ) were used. Results: They were obtained through Spearman's correlation coefficient, showing a highly significant relationship between the influence of the aesthetic model and body dissatisfaction ( $r h o=, 785 * *, p<0.000)$. By the same token, the relationship between the dimensions of influences of the aesthetic model and the variable of body dissatisfaction are significant. Conclusions: The greater the influence of the beauty model, the greater the concern will be for the body image and weight in adolescents.
\end{abstract}

Keyworks: Influence of aesthetic models, body dissatisfaccion, adolescents.

\footnotetext{
${ }^{1}$ Psicólogo, Servicio educacional hogar y salud. Asociación Peruana Central Este, Lima, Perú.

${ }^{2}$ Psicóloga en IADPA Librería. Alajuela, Costa Rica.

${ }^{3}$ Directora de la Unidad de Posgrado de Psicología, Escuela de Posgrado, Universidad Peruana Unión, Lima, Perú.
} 


\section{INTRODUCCIÓN}

En el trascurso de los años los cánones de belleza han cambiado de manera drástica, ya que la sociedad enaltece el prototipo de belleza en personas delgadas $\mathrm{y}$ mantiene prejuicios hacia la figura gruesa. Esto provoca en las personas insatisfacción con respecto a su cuerpo, pero cuando este problema se agrava puede derivar en trastornos alimenticios tales como anorexia o bulimia (Castiglia, 2015).

A nivel mundial, la International Society of Aesthetic Plastic Surgery (ISAPS, 2014) mencionó que la percepción de la imagen corporal se ve directamente influenciada por el modelo estético. Según las estadísticas se realizan más de 20 millones de intervenciones estéticas en todo el mundo; en Corea del Sur, una de cada 600 personas se hace injertos de grasa; asimismo, en Estados Unidos, una de cada 1000 se aumenta los senos; de la misma forma en Colombia, una de cada 2300 se aumenta los glúteos. En Perú, el Instituto Nacional de Salud Mental "Honorio Delgado-Hideyo Noguchi" INSM "HDHM” (2013) reportó en un estudio epidemiológico que el $7,4 \%$ de los adolescentes se siente nada o poco satisfecho con su aspecto físico. Además, el 0,2\% presente anorexia, el $0.3 \%$, bulimia y el $4,4 \%$, deseos suicidas relacionados con la autoimagen.

Las variables estudiadas, en esta investigación, son analizadas generalmente de manera independiente y con otros constructos, como por ejemplo; problemas de conducta alimentarias, índice de masa corporal, estado nutricional y afecto positivo (Miraval et al. 2014; Benel, Campos y Cruzado, 2012; Prieto y Muñoz, 2015). Por otra parte, resulta difícil delimitar el concepto de influencias del modelo estético, a causa de la falta de artículos teóricos que aborden el tema. Además, con respecto a la población, estas variables no han sido estudiadas en instituciones con principios religiosos, como los colegios católicos, evangélicos, mormones, adventistas, entre otros. Se considera específicamente estas poblaciones, pues es posible que la educación cristiana permita que los adolescentes perciban de distinta forma los modelos estéticos y la influencia de estos. Asimismo, estas variables no son ampliamente estudiadas en muestras clínicas, hecho que permitiría una mayor comprensión de estas y de cómo, en su estado más grave, pueden desencadenarse en trastornos, como por ejemplo los alimenticios y dismórficos.

En los últimos años, a causa del incremento de estos trastornos, existe mayor interés para estudiar las influencias del modelo estético e insatisfacción corporal, de modo que estos términos están siendo más utilizados por los profesionales de la salud mental. Es así que Carrillo y Del Moral (2013) mencionaron que las influencias del modelo estético son la construcción social de los cánones de belleza que varían en cada cultura y que influyen, en las percepciones del propio cuerpo. Por otro lado, Espina, Asunción, Ochoa, Yenes y Alemán (2001) definieron a la insatisfacción corporal como la desvalorización y preocupación exagerada del propio cuerpo, que comprende aspectos perceptuales, cognitivos y afectivos. Además, según Baile, esta surge de la discrepancia entre la imagen corporal real y la ideal (citado por Lúcar, 2012).

Habiendo señalado y observado la carencia de diversidad en el estudio de estas variables, el estudio pretende determinar si existe relación significativa entre la influencia de los modelos estéticos e insatisfacción corporal en una población de mujeres adolescentes.

\section{MATERIALES Y MÉTODOS}

El estudio es de diseño no experimental, de corte transversal y de alcance correlacional. Para la selección de los participantes se empleó el método no probabilístico, de esta manera se contó con un total de 255 estudiantes mujeres que cursaban del $3^{\circ}$ al $5^{\circ}$ año de educación secundaria de la Institución Educativa Manuel Gonzáles Prada, Huaycán-Ate con un rango de edades entre 13 y 17 años. A cada una de ellas se le solicitó su consentimiento como participantes voluntarios en la investigación.

El $72,9 \%$ de las evaluadas se encuentran entre las edades de 15 a 17 años. Asimismo, el 60,8\% de las evaluadas presentan un peso de $46 \mathrm{~kg}$ a $55 \mathrm{~kg}$ y alcanzan una talla de entre $1.51 \mathrm{~m}$ y $1.60 \mathrm{~m}(49 \%)$. Con respecto al grado de instrucción, el 35,3\% cursa el $4^{\circ}$ año de educación secundaria. Por otro lado, el $54,1 \%$ pertenece a la religión católica. 
Influencia del modelo estético e insatisfacción corporal en adolescentes mujeres de una institución educativa estatal de Lima este, 2016

Tabla 1

Características sociodemográficas de las participantes

\begin{tabular}{cccc}
\hline Datos Sociodemográficos & Categoría & N & $\%$ \\
\hline Edad & $13-14$ & 69 & 27,1 \\
& $15-17$ & 186 & 72,9 \\
Peso & $45 \mathrm{~kg}$ a menos & 52 & 20,4 \\
& $46 \mathrm{~kg} \mathrm{a} 55 \mathrm{~kg}$ & 155 & 60,8 \\
& $56 \mathrm{~kg}$ a $65 \mathrm{~kg}$ & 43 & 16,9 \\
& $66 \mathrm{~kg}$ a más & 5 & 2,0 \\
Talla & $1.40 \mathrm{~m}$ a $1.50 \mathrm{~m}$ & 111 & 43,5 \\
& $1.51 \mathrm{~m}$ a $1.60 \mathrm{~m}$ & 125 & 49,0 \\
Procedencia & $1.61 \mathrm{~m}$ a más & 19 & 7,5 \\
& Costa & 172 & 67,5 \\
& Sierra & 62 & 24,3 \\
Grado de instrucción & Selva & 21 & 8,2 \\
& Tercero & 89 & 34,9 \\
& Cuarto & 90 & 35,3 \\
\multirow{2}{*}{ Religión } & Quinto & 76 & 29,8 \\
& Evangélico & 66 & 25,9 \\
& Católico & 138 & 54,1 \\
& Otros & 51 & 20,0 \\
\hline
\end{tabular}

\section{Instrumentos}

Para el estudio se utilizó el Cuestionario de Influencias del Modelo Estético (CIMEC) construido en España por Toro, Salamero y Martínez (1994), cuya finalidad fue medir las influencias culturales relevantes que contribuyen a provocar, facilitar o justificar el adelgazamiento, en especial por razones estéticas y sociales. Fue adaptado a Perú por Castiglia (2015), constituido por 32 ítems y conformado de 4 dimensiones: influencia de la publicidad (11 ítems), angustia por la imagen corporal (8 ítems), influencia de modelos y situaciones sociales ( 9 ítems) e influencia de los mensajes verbales (4 ítems). Para el presente estudio se comprobó las propiedades psicométricas del cuestionario. Respecto a la fiabilidad global, se obtuvo un coeficiente alpha de Cronbach adecuado (926); asimismo, se realizó la validez del constructo a través de la correlación $r$ de Pearson, donde las dimensiones están correlacionadas significativamente, además de la validez de contenido por criterio de jueces.
Por otro lado, para medir la variable insatisfacción corporal, se utilizó el Body Shape Questionnaire (BSQ) creado por Cooper, Taylor y Fairbun (1987) compuesto por 34 ítems. Fue adaptado a Colombia por Castrillón, Luna, Avendaño y Pérez (2007) con el objetivo de medir la preocupación por el peso y la imagen corporal, la insatisfacción corporal, el deseo de perder, el miedo a engordar, la autodesvalorización debido a la apariencia y la evitación de situaciones donde el cuerpo pueda ser analizado. Además, está integrado por dos factores: insatisfacción corporal (20 ítems) y preocupación por el peso (14 ítems). Para la investigación, se realizó el análisis de fiabilidad en el que se obtuvo ,959 y la validez de constructo por medio de $\mathrm{r}$ de Pearson y contenido mediante el criterio de jueces.

Para el análisis de los datos se utilizó el programa estadístico SPSS 22, también, la prueba estadística Kolmogorov-Smirnov (K-S) para encontrar si existe una distribución normal de la muestra y la prueba Rho de Spearman para hallar la correlación de las variables. 


\section{RESULTADOS}

Tabla 2

Prueba de bondad de ajuste a la curva de normalidad para las variables de estudio.

\begin{tabular}{|c|c|c|c|c|c|}
\hline Instrumentos & Variables & Media & D.E. & K-S & $\mathbf{P}$ \\
\hline \multirow{5}{*}{$\begin{array}{l}\text { Influencia de los } \\
\text { modelos estéticos }\end{array}$} & Influencia global & 18.81 & 11.29 & .112 &, $000^{\circ}$ \\
\hline & Influencia de la publicidad & 5.23 & 4.63 & .133 &, $000^{\circ}$ \\
\hline & Angustia por la imagen corporal & 4.80 & 3.34 & .128 &, $000^{\circ}$ \\
\hline & $\begin{array}{l}\text { Influencia de modelos y } \\
\text { situaciones } \\
\text { sociales }\end{array}$ & 5.69 & 3.57 & .102 &, $000^{\circ}$ \\
\hline & Influencia de mensajes verbales & 3.09 & 1.72 & .138 &, $000^{\circ}$ \\
\hline $\begin{array}{l}\text { Insatisfacción } \\
\text { corporal }\end{array}$ & Insatisfacción global & 70.75 & 28.27 & .161 &, $000^{\circ}$ \\
\hline
\end{tabular}

Se observa en la Tabla 2 la prueba bondad de ajuste de Kolmogorov-Smirnov (K-S). Según los datos correspondientes a las variables de estudio, no presenta una distribución normal dado que el coeficiente obtenido (K-S) es significativo ( $\mathrm{p}<0.05$ ). Por lo tanto, para el análisis de correlación correspondiente se empleará estadística no paramétrica.

Tabla 3

Coeficiente de correlación entre las influencias del modelo estético e insatisfacción corporal.

\begin{tabular}{ccc}
\hline \multirow{2}{*}{ Influencias del modelo estético } & \multicolumn{2}{c}{ Insatisfacción corporal } \\
\cline { 2 - 3 } Influencia global &, $785^{* *}$ & $\mathrm{P}$ \\
\hline Influencia de la publicidad &, $629^{* *}$ &, 000 \\
Angustia por la imagen corporal &, $757^{* *}$ &, 000 \\
Influencia de modelos y situaciones sociales &, $683^{* *}$ &, 000 \\
Influencia de mensajes verbales &, $454^{* *}$ &, 000 \\
\hline
\end{tabular}

Por otro lado, en la tabla 3 se indica que existe relación altamente significativa entre nivel de influencia global e insatisfacción global $(\mathrm{r}=, 785 \mathrm{p}<0,01)$, de manera similar ocurre con las dimensiones de influencia de los modelos estéticos.

\section{DISCUSIÓN}

En cuanto a la relación entre las influencias del modelo estético y la variable insatisfacción corporal en adolescentes mujeres del $3^{\circ}$ a $5^{\circ}$ año de secundaria, el coeficiente rho de Spearman muestra un grado de intensidad de rho $=, 785^{* *}(\mathrm{p}<0,000)$ que indica una correlación altamente significativa. Esto quiere decir que, a mayor influencia del modelo de belleza, mayor será la preocupación por la imagen corporal y el peso en las adolescentes. Esto es coherente con la investigación de Esnaola, Rodríguez y Goñi (2010) quienes señalaron que los factores socioculturales, tales como los medios de comunicación, la familia y los grupos de pares, generan en el individuo, una valoración negativa del propio cuerpo. En este sentido, Martínez, Toro y Salamero (1996) afirmaron que las influencias del modelo estético se deben a factores socioculturales que contribuyen a la idea de tener una figura delgada. Asimismo, Salazar (2008) añadió que cada cultura impone sus propios estándares de belleza, ocasionando que las personas 
busquen alcanzar dicho ideal, debido a la necesidad de afiliación. En esta línea, Alegret, Comellas y Funes (2005) consideraron que la sociedad actual se caracteriza por el culto al cuerpo, en otras palabras, la preocupación exagerada del individuo por la figura corporal, que podría llevarlo a realizar conductas de riesgo. Una explicación de estos datos podría encontrarse en la teoría de la discrepancia, donde se analiza la imagen real y la ideal; la cual menciona que las personas que no cumplen con los criterios de belleza ideal, tendrían mayor disgusto hacia la imagen corporal y experimentarían sentimientos negativos (ansiedad, ira, tristeza), conductas de evitación, comprobación y purgación (Bermúdez-Bautista et al, 2009; Solano y Cano, 2010).

Respecto al análisis de la dimensión influencias de la publicidad y la insatisfacción corporal, se aprecia que las estudiantes con mayor interés hacia la publicidad de comerciales de televisión, reportajes, anuncios, relacionados al adelgazamiento o control de peso, tendrían mayor disgusto con la imagen corporal. Por ello, el coeficiente rho de Spearman mostró un grado de intensidad de rho $=, 629^{* *}, \mathrm{p}<0,000$, lo que evidencia que existe relación significativa alta entre la dimensión y la variable. Además, la constante exposición a campañas publicitarias, incrementa el deseo de las mujeres en alcanzar el prototipo de belleza vigente, debido a que muestran modelos delgadas. Esto sumado a la abstracción del fondo de la publicidad, lograría mayor identificación por parte de las lectoras, ya que resalta los beneficios y hace el mensaje más memorable y duradero (Lúcar, 2012 \& Salazar, 2007). En el mismo sentido, Bazán (2010) mencionó a la publicidad como la parte central en la promoción del ideal de belleza, ya que brinda relevancia a la estética corporal y la transmisión de mensajes que parecen alcanzables; sin embargo, ocasiona consecuencias negativas para la salud y la alteración afectiva con la imagen corporal.

Asimismo, en la dimensión angustia por la imagen corporal y la insatisfacción corporal, se muestra que las adolescentes sentirán mayor ansiedad al enfrentarse a situaciones que cuestionan el propio cuerpo y el deseo de ser iguales a los modelos sociales de delgadez, cuando manifiesten mayor descontento con la imagen corporal, puesto que el coeficiente de rho de Spearman evidencia un grado de rho $=, 757^{* *}$, $\mathrm{p}<0,000$, el cual indica que existe relación altamente significativa. En este sentido, las adolescentes sienten angustia o molestia cuando escuchan comentarios, tales como "llenita", "gordita", "redondita", aunque la intención del comentario sea positiva, o evitan situaciones que perciben o interpretan como amenazantes, como, por ejemplo, usar ropa de baño o ropa más corta en temporada de verano (Virues, 2005). Por otro lado, Toro (citado por Mancilla y Gómez, 2006) señala que la exposición de mujeres a imágenes que muestren la figura ideal, ocasiona angustia por la imagen corporal o estados de ánimo negativos. Estos resultados muestran que los modelos de belleza son demasiado elevados y difíciles de alcanzar para las mujeres; esto genera preocupación y angustia por no cumplir con los estándares sociales de belleza, lo que ocasiona a su vez insatisfacción por su imagen corporal (Webster y Tiggeman, 2010).

Del mismo modo, se encontró relación altamente significativa entre las influencias de modelo y situaciones sociales e insatisfacción corporal (rho= $\left., 683^{* *} \mathrm{p}<0,000\right)$. Lo cual indica que las adolescentes que tienen mayor interés hacia los agentes y situaciones que transmiten los modelos estéticos, tales como las anfitrionas que aparecen en las pasarelas o en los anuncios de ropa, etc., experimentan mayores niveles de sentimientos negativos hacia el propio cuerpo. Este dato fue corroborado por Tagre (2010) quien sostuvo que la familia y las personas cercanas (tíos, primos) ejercen influencia en el control del peso, debido a que las adolescentes mencionan haber recibido comentarios respecto a la alimentación; por ejemplo, "estás comiendo demasiado, ya no comas", "ya vas por la segunda tortilla, qué gorda" entre otros. En la misma línea, Rivaola y Penna (2006) sostuvieron que los cuerpos de las mujeres en fotografías o anuncios de jeans, o ropa interior, ocasionan drásticamente la aparición de signos de conductas y actitudes anómalas como la obsesión por la delgadez, lo que está relacionado con sentimientos de inseguridad con la autoevaluación de su imagen corporal. Finalmente, Bandura (1977) mencionó que la presión de los grupos de pares, hará que las adolescentes estén más vulnerables a conductas alimentarias poco saludables y a la influencia de los modelos sociales, vinculados al ideal de delgadez, a través del aprendizaje observacional.

Por otro lado, en los resultados entre influencia de los mensajes verbales e insatisfacción corporal, se encontró una relación altamente significativa entre las variables mencionadas $(\mathrm{rho}=, 454 * * \mathrm{p}<0,000)$. Esto quiere decir que, a mayor preocupación por la imagen corporal, mayor es el interés hacia artículos, reportajes, libros y conversaciones relacionados con la delgadez. En este sentido, los mensajes transmitidos por los medios de comunicación, cobran relevancia en la adolescencia, ya que en esta etapa surgen diversos cambios, que provocan dudas, miedo, ansiedad, generando la necesidad de ser valorados y aceptados por su entorno (Salazar, 2008). Asimismo, Tagre (2010) mencionó que los temas de conversación 
del género femenino en esta etapa, están referidos al ejercicio físico o conseguir un cuerpo delgado, dietas, comentarios acerca de que opinan los hombres en relación al cuerpo de las mujeres, etc. Ante ello, se concluye que la apariencia física en la adolescencia es muy importante; mientras las mujeres desean tener una figura corporal delgada, aun si están en un peso apropiado, los varones están preocupados por ganar masa muscular (Ramos, Rivera de los Santos y Moreno, 2010).

El estudio tiene como fortalezas, en primer lugar, su aporte para el incremento del conocimiento sobre la relación entre influencias del modelo estético y la insatisfacción con la imagen corporal. En segundo lugar, el hecho de haber realizado la investigación en una zona distinta, como es Lima este y brindar una línea base de la problemática, de modo que el centro educativo e instituciones aledañas tomen las medidas adecuadas. Sin embargo, también se señala las limitaciones del presente estudio, con una muestra pequeña y constituida solamente por mujeres, lo cual limita los resultados en la población de interés.

Por lo tanto, se recomienda que las futuras investigaciones realicen estudios en una muestra más amplia o en distintas poblaciones, es decir, poblaciones constituidas por personas de otro sexo (varones) de edades inferiores, pertenecientes a instituciones con principios religiosos y pertenecientes a muestras clínicas. Asimismo, se recomienda considerar otras variables que puedan incidir en la insatisfacción corporal de las adolescentes, tales como autoconcepto, autoestima, bienestar psicológico y estilos de crianza.

\section{Declaración de financiamiento y de conflicto de intereses:}

El estudio fue financiado por los autores, quienes declaran no tener algún tipo de conflicto de interés en la investigación realizada.

\section{Correspondencia:}

Betsaida Barja Becerra

Servicio educacional Hogar y salud. IADPA Librería. Alajuela, Costa Rica

e-mail: lizet.barja@gmail.com

\section{REFERENCIAS BIBLIOGRÁFICAS}

Alegret, J., Comellas, J. \& Funes, J (2005). Adolescentes: Relaciones con los padres, drogas, sexualidad $y$ culto al cuerpo. Barcelona: Lección Familia y Educación. Recuperado de: https://books.google.com/ books?isbn $=8499802419$

Bandura, A. (1977). Social Learning Theory. New York: General Learning Press

Bazán, C. (2010). Detección en mujeres argentinas de la correlación ilusoria "a mayor delgadez, mayor salud". Sesgos de atribución y factores psico-sociales asociados (Tesis Doctoral, Universidad de Palermo). Recuperado de: dspace.palermo.edu/dspace/handle/10226/1663

Bermúdez-Bautista, S., Franco-Paredes, K., ÁlvarezRayón, G., Vázquez-Arévalo, R., López-Aguilar, X., Téllez-Girón, M., Amaya-Hernández, A. \& MancillaDíaz, J (2009). El rol de la insatisfacción corporal e influencia del grupo de pares sobre la influencia de la publicidad, los modelos estéticos y dieta. Revista Mexicana de Investigación en Psicología, 1(1), 9-18. Recuperado de: http://new.medigraphic.com/cgi-bin/ resumen.cgi?IDARTICULO $=40467$

Castiglia, M. (2015). Adaptación del cuestionario de influencias del modelo estético corporal en adolescentes limeñas. (Tesis de Licenciatura, Pontifica Universidad Católica del Perú). Recuperado de:http://tesis.pucp.edu.pe/repositorio/bitstream/ handle/123456789/6535/CASTIGLIA FRANCO MARIA_ADOLESCENTES_LIMENAS . pdf? sequence $=1$

Castrillón, D.; Luna, I.; Avendaño, G. \& Pérez, A. (2007). Validación del Body Shape Questionnaire (Cuestionario de la Figura Corporal) BSQ para la población colombiana. Acta Colombiana de Psicología, 10(1), 15-23. Recuperado de http://www.scielo.org.co/pdf/ acp/v10n1/v10n1a03.pdf

Cooper, P., Taylor, M., Cooper, Z. \& Fairburn, C. (1987). The development and validation of the Body Shape Questionnaire. International Journal Eating Disorders, 6(1), 485-494. Recuperado de: http://onlinelibrary.wiley. com/doi/10.1002/1098-108X(198707)6:4\%3C485::AIDEAT2260060405\%3E3.0.CO;2-O/pdf

Esnola, I., Rodríguez, A. \& Goñi, A (2010). Body dissatisfaction and perceived sociocultural pressures: gender and age differences. Revista Salud Mental,. 33(1), 21- 29. Recuperado de: new.medigraphic.com/ cgi-bin/resumen.cgi?IDARTICULO=24238

Lúcar, M. (2012). Insatisfacción con la imagen corporal e influencias del modelo estético de delgadez en un grupo de adolescente mujeres. (Tesis de licenciatura, Pontifica Universidad Católica del Perú). Recuperado de:http://tesis.pucp.edu.pe/repositorio/bitstream/ handle/123456789/1723/LUCAR_FLORES_MIRIAM_ INSATISFACCION_MUJERES.pdf? sequence $=1$ 
Mancilla, J. \& Gómez, G. (2006). Trastornos alimentarios en Hispanoamerica. México: El Manual Moderno.

Martínez, E., Toro, J. \& Salamero, M. (1996). Influencias socioculturales favorecedoras del adelgazamiento y sintomatología alimentaria. Revista de Psiquiatría de la Facultad de Medicina de Barcelona, 23(5), 125-133.

Ramos, P., Rivera, F., \& Moreno, C. (2010). Diferencias de sexo en imagen corporal, control de peso e ídice de masa corporal de los adolescnetes españoles. Psicothema, 22(1), 77-83. Recuperado de: http://www. psicothema.com/pdf/3699.pdf

Rivaola, M. \& Penna, F. (2006). Los factores socioculturales y su relación con los trastornos alimentarios e imagen corporal. Revista intercontinental de psicología $y$ educación, 8(2), 6-12. Recuperado de: http://www. redalyc.org/pdf/802/80280205.pdf

Salazar, Z. (2007). Imagen corporal femenina y publicidad en revistas. Rev. Ciencias Sociales, 116(2), 71-85. Recuperado de: http://www.redalyc.org/pdf/153/15311605.pdf

Salazar, Z. (2008). Adolescencia e imagen corporal en la época de la delgadez. Revista Reflexiones, 87(2), 67-80. Recuperado de: www.redalyc.org/articulo. oa? $\mathrm{id}=72912555004$

Solano, N. \& Cano, A. (2010). IMAGEN: Evaluación de la Insatisfacción con la Imagen Corporal. Madrid: TEA.
Tagre, C. (2014). Influencia del modelo estético corporal en las estudiantes de Bachillerato de un colegio privado del municipio da Santa Catarina Pínula. (Tesis de grado, Universidad Rafael Landivar). Recuperado de: http://recursosbiblio.url.edu.gt/tesiseortiz/2014/05/42/ Tagre-Carlos.pdf

Toro, J., Salamero, M. \& Martínez, F. (1994). Assessment of sociocultural aesthetic body shape model in anorexia nervosa. Acta Psychiart Scond, 89(1), 147151. Recuperado de: http://onlinelibrary.wiley.com/ doi/10.1111/j.1600-0447.1994.tb08084.x/pdf

Virues, R. (2005, 25 de mayo). Estudio sobre ansiedad. Revista Psicología Cientifica.com, 7(8). Recuperado de: http://www.psicologiacientifica.com/ansiedad-estudio

Webster, J. \& Tiggemam, . (2010). The relationship between women's body satisfaction and self-image across the life span: the role of cognitive control. The journal of genetic psychology, 162(2), 241-252. Recuperado de: http://dx.doi.org/10.1080/00221320309597980.

Recibido: 20/02/2017

Aceptado: 15/04/2017 